\title{
Face And Palmprint Multimodal Biometric System Based On Bit-Plane Decomposition Approach
}

\author{
Therry Z. Lee, David B.L. Bong \\ Faculty of Engineering, Universiti Malaysia Sarawak, 94300 Kota Samarahan, Malaysia
}

\begin{abstract}
Bit-plane decomposition approach has been introduced lately for single trait biometric system such as face, palmprint, and fingerprint recognition. This approach is able to provide promising high performance rate while reducing the data dimensionality. However, this approach has not been tested on multimodal biometric system which uses more than one biometric trait. Hence, this paper introduces a new multimodal biometric system based on face and palmprint fusion with bitplane decomposition approach. Pixel level fusion is applied by using simple averaging method before bit-plane feature extraction. Principal Component Analysis is also used on the hybrid face-palm bit planes for further dimension reduction before being classified by Feedforward Backpropagation Neural Network. The experimental results show that the proposed system is able to provide high recognition rate.
\end{abstract}

\section{INTRODUCTION}

When two or more biometrics information are consolidated in a system to form the identity of a person, it is known as a multibiometric system. This system is able to provide an extra level of assurance for identification. Besides the virtue of higher accurateness, it alleviates several limitations of single modal biometric system such as non-universality issue. Many techniques have been applied on creating fusion between biometrics to form various types of multimodal biometric system. There are several levels of fusion such as pixel level, feature level, score level, and decision level. Most researches were conducted for feature and score level fusions, while only partial studies were done on the integration of face and palmprint images at pixel level fusion. Furthermore, implementation of face and palmprint fusion based on the recently introduced bit-plane extraction technique has yet to be deployed in a multimodal biometric system.

Previous researchers have introduced bit-plane decomposition approach for face, palmprint and fingerprint recognition respectively [1]-[3]. The bit-plane decomposition approach is able to provide promising high performance rate while reducing the data dimensionality. It is based on the assumption where the higher order of decomposed bit planes contain more biometric feature, while the lower order of the bit planes consist less information of the biometric.

Therefore, this paper proposes a new multimodal biometric system based on bit-plane decomposition. Due to the availability of richer and significant information at pixel level[4], the proposed system is based on fusion of face and palmprint at pixel level with bit-plane feature extraction. For identity matching or classification, Principal Component Analysis (PCA) is used with Feedforward Backpropagation Neural Network.

\section{Proposed Multimodal Biometric System}

Fig. 1 shows the flowchart of the proposed system. Firstly, preprocessing is performed on the face and palmprint images by applying intensity adjustment to highlight the important details of the image. Both images are then consolidated at pixel level by using simple averaging method. Next, the bitplane of the newly hybrid image is extracted as feature input for classification. PCA is applied to reduce the dimensionality of the hybrid image which later being fed into neural network. The performance is evaluated by using recognition rate. The details of some stages are as follow.

\section{A. Pixel level fusion}

To consolidate the face and palmprint image, averaging method is used for pixel level fusion. It is a direct method where the value of the pixel of each image is obtained and added. The average is then calculated by dividing the sum with 2. The average value is the fusion result of each corresponding pixel of the hybrid image. This can be defined by,

$$
X(i, j)=\{F(i, j)+P(i, j)\} / 2
$$

Where $X(i, j)$ is the hybrid face and palm image, $F(i, j)$ is the face image and $P(i, j)$ is the palmprint image.

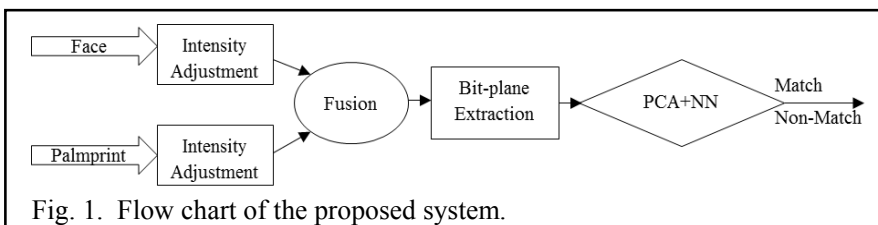

\section{B. Bit-plane Decomposition}

The feature extraction technique in the proposed system is the bit-plane decomposition approach. The idea of decomposing an image from gray-scale into a collection of binary images is introduced by T Schwarz in [5], which is called the bit-plane decomposition technique. An 8-bits gray level image has pixel values ranging from 0 to 225 . Therefore, it can be decomposed into 8 layers or bit-planes, whereby the first bit-plane (bit-plane 0) consists of the least significant bits while the last bit-plane (bit-plane 7) comprises the most significant bits.

The general derivation for bit-plane feature extraction is summarized as,

$$
f(x, y)=\left[\begin{array}{cccc}
f(0,0) & f(0,1) & \ldots & f(0, N-1) \\
f(1,0) & f(1,1) & \ldots & f(1, N-1) \\
\vdots & \vdots & \ldots & \vdots \\
f(M-1,0) & f(M-1,1) & \ldots & f(M-1, N-1)
\end{array}\right]
$$

